\title{
Point-to-Point Repetitive Control of Functional Electrical Stimulation for Drop-Foot
}

\author{
A. P. Page*, C. T. Freeman \\ Electronics and Computer Science, University of Southampton, Southampton, SO17 1BJ, UK
}

\begin{abstract}
Drop-Foot is a common problem resulting from a range of neurological conditions, and prevents normal leg swing during gait, leading to abnormal, inefficient motion with an increased risk of falling. It damages the quality of life of over 122,000 people in the US and 11,400 people in the UK every year.

Functional electrical stimulation (FES) addresses drop-foot by artificially contracting the tibialis anterior, and has had considerable success both clinically and commercially. However current commercial controllers are open loop and have long set-up times. The few controllers in the research domain are predominantly open-loop, lack accuracy, and struggle with muscle delays, non-linearities and the onset of fatigue. More advanced controllers require extensive sensor data and/or are highly dependent on an identified model. Recent developments have shown model based controllers combined with learning can facilitate higher accuracy, however previous attempts employed batch-wise learning, and led to disjointed control signals.

This paper applies repetitive control (RC) to drop-foot for the first time, facilitating a continuous, smooth process of learning with no resetting. To maximise performance, a comprehensive extension to the standard $\mathrm{RC}$ framework is undertaken to enable only isolated time points to be tracked, improving robustness and reducing memory and communication requirements. Experimental data confirms that RC can achieve normal gait when applied to FES-assisted gait with no voluntary effort. The new 'point-to-point' RC framework outperfrmed standard RC, while using only 5 data points per gait cycle and minimal control effort.
\end{abstract}

Keywords: Repetitive Control, Point-to-Point, FES, Gait, Assistive Technology, Drop Foot

\section{Introduction}

Drop-foot is characterised by ankle dorsiflexion weakness, caused by nerve or muscle damage. It results in abnormal gait, producing slow, tiring and often unsafe ambulation. Typically caused by compression or damage to the peroneal nerve, drop-foot manifests in approximately $20 \%$ of the 10 million people worldwide who survive stroke each year [1]. This number continues to climb due to increased 10 life expectancy and demographic changes. Additionally, multiple sclerosis (MS) can also lead to drop-foot, with studies showing that $63 \%$ of people with MS fear falling and $83 \%$ thus avoid activity 2. Full recovery from drop-foot if due to skeletal

\footnotetext{
${ }^{*}$ Corresponding author

Email addresses: app2g13@soton.ac.uk (A. P. Page), cf@ecs.soton.ac.uk (C. T. Freeman)
}

Preprint submitted to Journal of Control Engineering Practice
15 or neuropathy/neurological issues is often not possible, so there is an urgent need for effective technologies to aid mobility and combat pathological gait. Mechanical ankle orthoses are commonly used and comprise of a rigid or semi-rigid splint that fixes 20 the ankle. These typically restrict motion and can discourage recovery, leading to increased spasticity and soft tissue shortening. For this reason, clinicians are often unwilling to prescribe such devices, as reflected by studies in which $47 \%$ of people have 25 never used them 3 .

The leading active intervention technology is functional electrical stimulation (FES), and can be used if drop-foot is caused by damage to the central nervous system, leaving the peripheral nerves so intact. Small electrical impulses, via small wearable skin electrodes, allow FES to control muscle contraction. Clinical trials of FES have shown an increase in walking speed by $16 \%$, a reduction in

June 12, 2019 
effort of $29 \%$ as well as reduced spasticity, anxiety 35 and depression [4].

A variety of commercial FES systems for dropfoot exist, but all employ open-loop control which is usually triggered by a pressure sensor mounted in the heel or a button on a supporting frame. This 40 generates effective, but crude, dorsiflexsion around the ankle. In the research domain, a range of open and closed loop systems have been developed. The first approaches to open loop FES control required manual user input to initiate stimulation pulses 45 5. Manual user commands were compared with "hand-crafted" rules, and the latter found to improve walking speed by $7 \%$ and reduce the stimulation duration by $38 \%$. Open-loop control has also been used with an electrode array 6. Here electrode pads were sequentially stimulated to locate an optimal 'virtual' pad. However, once the optimal pad was found, a simple open loop ramp input was applied and was initialised with a heel switch.

Fuzzy logic and PID controllers have been com-

55 bined with neural networks in [7, and applied in a study with 3 hemiplegic participants. The experiment consisted of each participant lying on a bed with the ankle elevated off the bed, the controller ${ }_{110}$ then attempted to track a gait reference stretched 60 over 13 seconds and consisting of 500 points. The neural network achieved an angular root mean squared error (RMSE) of $8.11^{\circ}, 7.81^{\circ}$ and $5.59^{\circ}$ for each participant. Neural network combined with 115 PID performed better, achieving $5.57^{\circ}, 4.83^{\circ}$ and

$655.05^{\circ}$, but neural network combined with fuzzy logic performed best with $4.07^{\circ}, 3.75^{\circ}$ and $4.19^{\circ}$.

An adaptive approach based on sliding mode control and non-linear compensation controlled the agonist-antagonist muscles to generate ankle move70 ment in [8]. It considered each muscle as a subsystem and employed decentralized control. The study included experimental validation on 3 healthy and 3 paraplegic subjects. The experiment was conducted on a bench, with hip angle $90^{\circ}$, knee angle $0^{\circ}$, and 75 the ankle free to move. The references tracked, however, were not natural gait, rather a bipha- 125 sic trapezodial (20s period), biphasic raised-cosine (12s period) and sine wave (20s period). The mean RMSE for healthy patients, across all tests, was ${ }_{80} 3.2^{\circ} \pm 1.2^{\circ}$ and for paraplegic subjects $3.4^{\circ} \pm 0.2^{\circ}$.

The most advanced approaches reported used it- 130 erative learning control (ILC) 9, 10. The latter employed 6 inertial measurements to provide the ankle angle. Here the ILC activated after the heel ${ }_{85}$ was lifted and had a duration equal to the period of the swing phase. The ILC was then reset before the next cycle. A study with 6 post stroke patients validated the approach. Here the first cycle was an approximation of the required input. Although no data values were explicitly reported, graphs indicate RMSE values in the range $2^{\circ}$ to $6^{\circ}$ were achieved within 2 cycles and maintained. Although effective, the piecewise application of ILC introduced discontinuities in the input signals and resulting motion, as well as complicating design and convergence properties. In addition, it required significant sensor data and an accurate system model.

The above approaches firstly show the improvements possible by using closed-loop control combined with model based approaches to address drop-foot. Learning has then been found to further improve performance, taking advantage of the inherent cyclic nature of gait. However, the ILC methods used consider each step as a distinct, separate task rather than a constantly repeating process. This has degraded both performance and convergence properties. To address these limitations, this paper will develop a control framework that also embeds learning in a continuous manner, thereby avoiding discontinuities, and leading to transparent performance and convergence properties. Furthermore, it will also utilise minimal data, hence reducing communication and energy demands that are critical in wearable devices.

The approach will employ repetitive control (RC), and constitutes its first application for dropfoot. The next section develops the underlying theoretical background, which is then expanded to take a new 'point-to-point' form that embeds the minimal data requirement.

\section{Control Framework}

Iterative learning control (ILC) is applicable to systems undertaking repeated tasks, with the fundamental requirement that they reset to identical initial conditions between attempts. Since its inception in the 1980s, ILC has developed a mature theoretical background and has been applied to numerous fields. RC emerged as a method of controlling systems that track a periodic reference. However, no resetting is assumed, and this has resulted in a clear separation between disciplines in terms of both theoretical underpinning, and application areas. However, duality properties connect both ILC and $\mathrm{RC}$, and have been defined in [1]. 
$\mathrm{RC}$ is underpinned by the internal model principle, which states that a necessary condition for any system to reject a periodic disturbance is to have a model of that disturbance embedded within its control structure. RC was previously employed in FES tremor suppression in [12, where an underlying linear model was assumed. This was possible since linearising action was used to remove the non-linearities associated with FES induced limb dynamics. The approach of linear RC design, followed by linearising action will thereby be adopted in the current application to drop-foot.

Traditional RC structures enforce tracking of a reference at all time points. This requires full sensor data, and often leads to slow convergence and a lack of robustness to model uncertainty and noise. To address this drawback, the next section expands the $\mathrm{RC}$ framework to allow tracking of isolated points. This is the first RC framework to do this, although there exist parallel approaches in the frequency domain which track only a subset of frequencies [13].

\subsection{Repetitive Control Formulation}

Let plant $P$ be expressed by the state-space form

$$
P: u \mapsto y \quad\left\{\begin{aligned}
x_{P}(i+1) & =A_{P} x_{P}(i)+B_{P} u(i) \\
y(i) & =C_{P} x_{P}(i)+D_{P} u(i)
\end{aligned}\right.
$$

where the state transition matrix $A_{P} \in \mathbb{R}^{n_{P} \times n_{P}}$, the input matrix $B_{P} \in \mathbb{R}^{n_{P} \times m}$, the output matrix $C_{P} \in \mathbb{R}^{o \times n_{P}}$, the feed-through matrix $D_{P} \in \mathbb{R}^{o \times m}$. Here, $m$ is the number of inputs, $o$ is the number of outputs, $n_{P}$ is the number of states, $x_{P}$ is the state vector, $u(i)$ is the input and $y(i)$ is the output at sample $i \in \mathbb{N}$. Here, without loss of generality, $\left(A_{P}, B_{P}, C_{P}, D_{P}\right)$ are assumed minimal, and the ${ }_{170}$ inital state is $x_{P}(0)=x_{0}$.

Let reference signal $r$ be $N$-periodic, that is $r(i)=r(i+N)$ for a given $N \in \mathbb{N}$ and all $i$. The RC tracking problem is for the plant output to asymptotically converge to the reference as the cycle in$\operatorname{dex}, k \in \mathbb{N}$, increases. That is

$$
\lim _{k \rightarrow \infty} y(k N+i)=r(i), \quad i=0, \cdots, N-1
$$

while the control input converges to a fixed signal,

$$
\lim _{k \rightarrow \infty} u(k N+i)=\hat{u}(i), \quad i=0, \cdots, N-1 .
$$

Now introduce the one-sample advance operator $q$, defined by $q(v(k))=v(k+1)$ for any signal $v$.
The most common RC update form is then

$$
u(i+N)=Q(q)(u(i)+L(q) e(i)),
$$

where, $\forall i \in \mathbb{N}$, and $Q(q), L(q)$ are filters and $e(i)=$ $r(i)-y(i)$ is the tracking error, as seen in Fig. 1 . See [14] for an overview of RC design approaches.

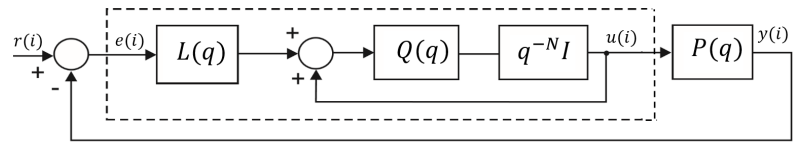

Figure 1: Traditional RC structure.

Defining $P(q)=C_{P}\left(I q-A_{P}\right)^{-1} B_{P}+D_{P}$, from (4) it follows that the disturbance-free control action evolution is

$$
\begin{aligned}
q^{N}(u(i)) & =u(i+N)=Q(q)(1-L(q) P(q)) u(i) \\
& +Q(q) L(q) r(i) .
\end{aligned}
$$

and a necessary and sufficient condition for convergence of the input is that the poles of the closed loop system

$$
\frac{u(i)}{r(i)}=\frac{Q(q) L(q)}{I-q^{-N} Q(q)(I-P(q) L(q)}
$$

are all inside the unit circle. A sufficient condition for this is given by

$$
\|Q(q)(I-P(q) L(q))\|_{\infty}<1
$$

or equivalently, in the frequency domain, by

$$
\sup _{\omega \in[0, \pi]}\left|Q\left(e^{j w}\right)\left(I-P\left(e^{j w}\right) L\left(e^{j w}\right)\right)\right|<1 .
$$

Note, for an arbitrary reference $r$, convergence to zero error requires $Q(q)=I$.

\subsection{Lifted Repetitive Control Formulation}

In order to expand RC to track only a subset of points of $r(t)$, it is necessary to adopt the 'lifted' framework, in which the signals for each period, $k \in \mathbb{N}$, are packaged together as vectors. Although commonly used in ILC, it was only recently introduced for RC in [11]. Accordingly, the input and output signals are defined as the super vectors

$$
\begin{aligned}
& \boldsymbol{u}(k)=[u(k N), u(k N+1), \cdots u((k+1) N-1)]^{\top}, \\
& \boldsymbol{y}(k)=[y(k N), y(k N+1), \cdots y((k+1) N-1)]^{\top} .
\end{aligned}
$$


where $\boldsymbol{u}(k) \in \mathbb{R}^{m N}$ and $\boldsymbol{y}(k) \in \mathbb{R}^{o N}$. Similarly, the reference and error are given as

$$
\begin{aligned}
\boldsymbol{r}(k)=\boldsymbol{r} & =[r(0), r(1), \cdots r(N-1)]^{\top} \in \mathbb{R}^{o N}, \\
\boldsymbol{e}(k) & =\boldsymbol{r}-\boldsymbol{y}(k)
\end{aligned}
$$

and the tracking requirement (2) becomes

$$
\lim _{k \rightarrow \infty} \boldsymbol{y}(k)=\boldsymbol{r} \text {. }
$$

Using these signals, RC update (4) is written as

$$
\boldsymbol{u}(k+1)=\boldsymbol{Q}(\boldsymbol{q})(\boldsymbol{u}(k)+\boldsymbol{L}(\boldsymbol{q}) \boldsymbol{e}(k)),
$$

where $\boldsymbol{Q}$ and $\boldsymbol{L}$ are lifted versions of the robustness 180 and learning filters respectively. The plant dynam-

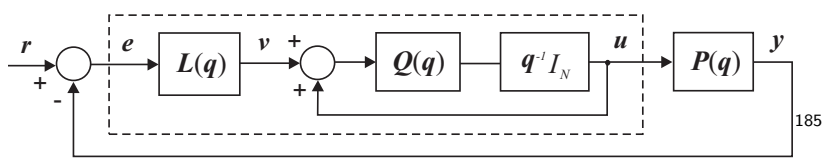

Figure 2: RC system lifted description.

ics (1) can be written in terms of the super-vectors (9), to yield the lifted plant dynamics

$$
\begin{aligned}
& \begin{aligned}
\underbrace{x_{P}((k+1) N)}_{\boldsymbol{x}_{P}(k+1)}= & \underbrace{A_{P}^{N} \underbrace{x_{p}(k N)}_{\boldsymbol{x}_{P}(k)}+}_{\boldsymbol{A}_{P}} \\
& \underbrace{\left[A_{P}^{N-1} B_{P}, A_{P}^{N-2} B_{P}, \cdots B_{P}\right]}_{\boldsymbol{B}_{P}} \boldsymbol{u}(k)
\end{aligned} \\
& \boldsymbol{P}:\left\{\begin{aligned}
\boldsymbol{y}(k)= & \underbrace{\left[\begin{array}{c}
C_{P} \\
C_{P} A_{P} \\
\vdots \\
C_{P} A_{P}^{N-1}
\end{array}\right]}_{\boldsymbol{C}_{P}} \underbrace{x_{P}(k N)}_{\boldsymbol{x}_{P}(k)}+ \\
& \underbrace{\left[\begin{array}{cccc}
D_{P} & 0 & \cdots & 0 \\
C_{P} B_{P} & D_{P} & \ddots & \vdots \\
\vdots & \ddots & \ddots & 0 \\
C_{P} A_{P}^{N-2} B_{P} & \cdots & C_{P} B_{P} & D_{P}
\end{array}\right]}_{\boldsymbol{D}_{P}} \boldsymbol{u}(k)
\end{aligned}\right.
\end{aligned}
$$

where $\boldsymbol{A}_{P} \in \mathbb{R}^{n_{P} \times n_{P}}, \boldsymbol{B}_{P} \in \mathbb{R}^{n_{P} \times m N}, \boldsymbol{C}_{P} \in$ $\mathbb{R}^{o N \times n_{P}}$ and $\boldsymbol{D}_{P} \in \mathbb{R}^{o N \times m N}$. Likewise, lifted forms of $\boldsymbol{Q}(\boldsymbol{q})$ and $\boldsymbol{L}(\boldsymbol{q})$ can be defined as

$\boldsymbol{Q}:\left\{\begin{aligned} \boldsymbol{x}_{Q}(k+1) & =\boldsymbol{A}_{Q} \boldsymbol{x}_{Q}(k)+\boldsymbol{B}_{Q}(\boldsymbol{v}+\boldsymbol{u})(k) \\ \boldsymbol{u}(k+1) & =\boldsymbol{C}_{Q} \boldsymbol{x}_{Q}(k)+\boldsymbol{D}_{Q}(\boldsymbol{v}+\boldsymbol{u})(k),\end{aligned}\right.$

where $\boldsymbol{A}_{Q} \in \mathbb{R}^{n_{Q} \times n_{Q}}, \boldsymbol{B}_{Q} \in \mathbb{R}^{n_{Q} \times m N}, \boldsymbol{C}_{Q} \in$ $\mathbb{R}^{m N \times n_{Q}}$ and $\boldsymbol{D}_{Q} \in \mathbb{R}^{m N \times m N}$, and

$$
\boldsymbol{L}:\left\{\begin{aligned}
\boldsymbol{x}_{L}(k+1) & =\boldsymbol{A}_{L} \boldsymbol{x}_{L}(k)+\boldsymbol{B}_{L} \boldsymbol{e}(k) \\
\boldsymbol{v}(k) & =\boldsymbol{C}_{L} \boldsymbol{x}_{L}(k)+\boldsymbol{D}_{L} \boldsymbol{e}(k),
\end{aligned}\right.
$$

where $\boldsymbol{A}_{L} \in \mathbb{R}^{n_{L} \times n_{L}}, \boldsymbol{B}_{L} \in \mathbb{R}^{n_{L} \times o N}, \boldsymbol{C}_{L} \in$ $\mathbb{R}^{m N \times n_{L}}$ and $\boldsymbol{D}_{L} \in \mathbb{R}^{m N \times o N}$. If $Q(q)$ and $L(q)$ have defined unlifted state-space forms, then explicit structured forms of 13 and $(14)$ can be defined, see 11 for details. Convergence condition (7) expressed for the lifted system corresponds to

$$
\|\boldsymbol{Q}(\boldsymbol{q})(I-\boldsymbol{P}(\boldsymbol{q}) \boldsymbol{L}(\boldsymbol{q}))\|_{\infty}<1,
$$

and it is shown in [11] that satisfying (7) and (8) is a sufficient condition for satisfying (15).

\subsection{Point-to-Point Repetitive Control}

Having introduced the lifted framework, the following section develops the framework necessary to enable RC to track a set of defined input positions at pre-described time points. Reducing the points in the control update reduces the data that need to be stored between periods, and also results in improved robustness and faster convergence.

Suppose a subset of $M<N$ points of reference $r$ are required to be tracked, with distinct indices

$$
i=i_{1}, i_{2}, \cdots, i_{M} \text {. }
$$

The subset of reference points can then be extracted from $\boldsymbol{r}$ using the projection operator $\boldsymbol{\Phi}: \mathcal{L}^{N m} \rightarrow$ $\mathcal{L}^{M m}$. For any lifted signal $s \in \mathcal{L}^{m N}$, let

$$
\begin{gathered}
\boldsymbol{\Phi}: \mathcal{L}^{m N} \rightarrow \mathcal{L}^{m M}: \boldsymbol{s} \mapsto \boldsymbol{s}^{\Phi}, \\
\boldsymbol{s}^{\Phi}(i)=\left[\begin{array}{c}
s\left(i N+i_{1}\right) \\
s\left(i N+i_{2}\right) \\
\vdots \\
\left.s\left(i N+i_{M}\right)\right)
\end{array}\right]=\overline{\mathbf{\Phi}} \boldsymbol{s}(i) \in \mathbb{R}^{m M},
\end{gathered}
$$

where

$$
\overline{\mathbf{\Phi}}_{j, i}=\left\{\begin{array}{ll}
I, & \text { if } i=i_{j} \\
0, & \text { otherwise }
\end{array} .\right.
$$

In particular, applying $\boldsymbol{\Phi}$ to $\boldsymbol{r}$ extracts the set of desired point-to-point output positions to be tracked

$$
\boldsymbol{r}^{\mathbf{\Phi}}=\boldsymbol{\Phi} \boldsymbol{r}=\left[\begin{array}{c}
r\left(i_{1}\right) \\
r\left(i_{2}\right) \\
\vdots \\
r\left(i_{M}\right)
\end{array}\right] .
$$

Given this new tracking objective, the RC system is now reformed as in Fig. 3. Error $\boldsymbol{e}$ is replaced by $\boldsymbol{\Phi} \boldsymbol{e}=\boldsymbol{e}^{\Phi}$, and now $\boldsymbol{Q}(\boldsymbol{q})$ and $\boldsymbol{L}(\boldsymbol{q})$ must be redesigned for this lower dimensional system. The tracking objective 11 is replaced by

$$
\lim _{k \rightarrow \infty} \boldsymbol{y}^{\Phi}(k)=\boldsymbol{r}^{\Phi}
$$




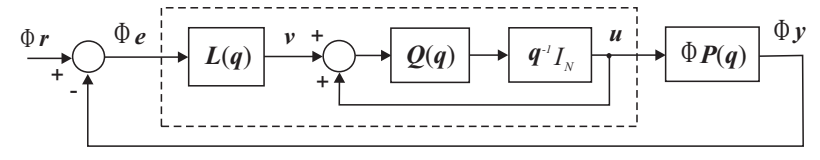

Figure 3: Point-to-point RC system lifted structure.

and the requirement (3) that the input converges to a fixed signal is now expressed as

$$
\lim _{k \rightarrow \infty} \boldsymbol{u}(k)=\hat{\boldsymbol{u}}
$$

A new RC update to solve the point-to-point problem can now be stated as follows.

Theorem 1. The general lifted $R C$ update form

$$
\boldsymbol{u}(k+1)=\boldsymbol{Q}(q)\left(\boldsymbol{u}(k)+\boldsymbol{L}(\boldsymbol{q}) \boldsymbol{e}^{\Phi}(k)\right)
$$

where $\boldsymbol{e}^{\Phi}(k)=\boldsymbol{r}^{\Phi}-\boldsymbol{\Phi} \boldsymbol{y}(k)$, and $\boldsymbol{L}(\boldsymbol{q}): \mathcal{L}^{m M} \rightarrow$ $\mathcal{L}^{m N}$ is selected to satisfy

$$
\|\boldsymbol{Q}(q)(I-\boldsymbol{\Phi} \boldsymbol{P}(\boldsymbol{q}) \boldsymbol{L}(\boldsymbol{q}))\|_{\infty}<1,
$$

solves the point-to-point RC problem (20), (21).

Proof. The lifted closed loop system is

$\boldsymbol{u}(i)=\boldsymbol{Q}(q) \boldsymbol{L}(q)\left(\boldsymbol{I}-q^{-1} \boldsymbol{Q}(q)(I-\boldsymbol{\Phi} \boldsymbol{P}(q) \boldsymbol{L}(q))^{-1} \boldsymbol{r}(i)\right.$

and from the internal model principle, 20, 21 hold true if its poles are all inside the unit circle. A sufficient condition for this is 23 .

The general lifted update 22 has a form which makes it difficult to choose $\boldsymbol{L}(\boldsymbol{q})$ transparently. The next theorem shows how it can be selected by modifying any of the many existing operators that have been proposed in traditional RC.

Theorem 2. Let unlifted operator $\bar{L}(q)$ satisfy the traditional $R C$ criterion

$$
\|I-P(q) \bar{L}(q)\|_{\infty}<1,
$$

or alternatively

$$
\sup _{\omega \in[0, \pi]}\left|I-P\left(e^{j w}\right) \bar{L}\left(e^{j w}\right)\right|<1,
$$

then the lifted operator

$$
\boldsymbol{L}(\boldsymbol{q})=\overline{\boldsymbol{L}}(\boldsymbol{q}) \boldsymbol{\Phi}^{*},
$$

satisfies condition (23) where $(\cdot)^{*}$ denotes the adjoint of its argument.
Proof. Inserting form 27 into 23) gives

$\left\|I-\boldsymbol{\Phi} \boldsymbol{P}(\boldsymbol{q}) \overline{\boldsymbol{L}}(\boldsymbol{q}) \boldsymbol{\Phi}^{*}\right\|_{\infty}=\left\|\boldsymbol{\Phi}(I-\boldsymbol{P}(\boldsymbol{q}) \overline{\boldsymbol{L}}(\boldsymbol{q})) \boldsymbol{\Phi}^{*}\right\|_{\infty}$,

and since (7), 26 imply

$$
\|I-\boldsymbol{P}(\boldsymbol{q}) \overline{\boldsymbol{L}}(\boldsymbol{q})\|_{\infty}<1
$$

it follows that

$$
\left\|I-\boldsymbol{\Phi} \boldsymbol{P}(\boldsymbol{q}) \overline{\boldsymbol{L}}(\boldsymbol{q}) \boldsymbol{\Phi}^{*}\right\|_{\infty} \leq\|I-\boldsymbol{P}(\boldsymbol{q}) \overline{\boldsymbol{L}}(\boldsymbol{q})\|_{\infty}<1,
$$

200 so that 23 holds with $\boldsymbol{Q}(\boldsymbol{q})=I$.

To illustrate Theorem 2, consider the so-called gradient RC update, proposed in [15, 16]. This is considered due to its track record in experimental effectiveness, attractive robustness properties, and ease of implementation. These were illustrated in, for example, its use in tremor suppression [12].

Lemma 3. The gradient $R C$ algorithm, given by

$$
\bar{L}(q)=\beta P^{*}(q),
$$

where $P^{*}(q)$ is the adjoint of $P(q)$ and $\beta$ is a scalar such that

$$
0<\beta<\frac{2}{\sup _{\omega \in[0, \pi]}\left|P\left(e^{j \omega}\right)\right|^{2}},
$$

satisfies the traditional $R C$ conditions 25) and (26). Note that, for causality of the $R C$ update, it is necessary that the plant satisfies the condition

$$
C_{P} A_{P}^{i} B_{P}=0 \quad \forall \quad i \geq N .
$$

Lemma 3 and Theorem 2 immediately yield a point-to-point RC update that satifies Theorem 1 . This lifted gradient point-to-point RC update is

$$
\boldsymbol{u}(k+1)=\boldsymbol{u}(k)+\beta(\boldsymbol{\Phi} \boldsymbol{P}(\boldsymbol{q}))^{*} \boldsymbol{e}^{\Phi}(k) .
$$

The next section focuses on computing the converged input limit $\hat{\boldsymbol{u}}$ within (21). This will show that one of the properties of the gradient point-toRC update is that the converged input signal optimises an objective function.

\subsection{Convergence to optimal input signal}

Theorem 4. Let gradient point-to-point $R C$ update (34) be modified to assume the more general form

$$
\boldsymbol{u}(k+1)=\boldsymbol{u}(k)+\underbrace{\beta \boldsymbol{F}(q) \boldsymbol{F}^{*}(q)(\boldsymbol{\Phi} \boldsymbol{P}(\boldsymbol{q}))^{*}}_{\boldsymbol{L}(\boldsymbol{q})} \boldsymbol{e}^{\Phi}(k)
$$


where $\boldsymbol{F}(\boldsymbol{q})$ is a lifted operator with unlifted statespace matrices $\left\{A_{F}, B_{F}, C_{F}, D_{F}\right\}$ satisfying

$$
C_{F} A_{F}^{i} B_{F}=0 \quad \forall \quad i \geq n_{F},
$$

with $n_{F}+n_{P} \leq N$, and $\beta$ a scalar satisfying

$$
0<\beta<\frac{2}{\sup _{\omega \in[0, \pi]}\left|P\left(e^{j \omega}\right)\right|^{2}\left|F\left(e^{j \omega}\right)\right|^{2}} .
$$

This solves point-to-point RC problem (20), (21). In particular, the converged control signal $\hat{\boldsymbol{u}}$ in (21) is the solution to the optimal control problem

$$
\hat{\boldsymbol{u}}:=\min _{\boldsymbol{u}}\|\boldsymbol{H}(\boldsymbol{q}) \boldsymbol{u}\|^{2} \quad \text { such that } \quad \boldsymbol{r}^{\Phi}=\boldsymbol{\Phi} \boldsymbol{P}(\boldsymbol{q}) \boldsymbol{u}
$$

where operator $\boldsymbol{H}(\boldsymbol{q})$ has a steady-state value equal to the inverse of the steady state of $\boldsymbol{F}(\boldsymbol{q})$.

Proof. The steady-state solution satisfies $\boldsymbol{u}(k)=$ $\hat{\boldsymbol{u}} \forall k$, so the operators can hence be replaced by

$$
\boldsymbol{P}=\left(\boldsymbol{D}_{P}+\boldsymbol{C}_{P} \boldsymbol{B}_{P}\right)
$$

Similarly for $\boldsymbol{H}$, with lifted state-space matrices $\left\{\boldsymbol{A}_{H}, \boldsymbol{B}_{H}, \boldsymbol{C}_{H}, \boldsymbol{D}_{H}\right\}$, we have in steady-state

$$
\boldsymbol{H}=\left(\boldsymbol{D}_{H}+\boldsymbol{C}_{H} \boldsymbol{B}_{H}\right)
$$

Using Lagrangian multiplier $\lambda$, the solution $\hat{\boldsymbol{u}}$, to (38) is

$$
\begin{aligned}
& \operatorname{argmin}\left\{J(\boldsymbol{u}), J(\boldsymbol{u})=\frac{1}{2}\left(\left(\boldsymbol{D}_{H}+\boldsymbol{C}_{H} \boldsymbol{B}_{H}\right) \boldsymbol{u}\right)^{\top}\right. \\
& \left.\times\left(\boldsymbol{D}_{H}+\boldsymbol{C}_{H} \boldsymbol{B}_{H}\right) \boldsymbol{u}+\lambda^{\top}\left(\boldsymbol{\Phi}\left(\boldsymbol{D}_{P}+\boldsymbol{C}_{P} \boldsymbol{B}_{P}\right) \boldsymbol{u}-\boldsymbol{r}^{\Phi}\right)\right\}
\end{aligned}
$$

and, since 40 is full rank, $\lambda$ satisfies

$$
\left(\boldsymbol{D}_{H}+\boldsymbol{C}_{H} \boldsymbol{B}_{H}\right)^{\top}\left(\boldsymbol{D}_{H}+\boldsymbol{C}_{H} \boldsymbol{B}_{H}\right) \hat{\boldsymbol{u}}+(\boldsymbol{\Phi} P)^{\top} \lambda=0
$$

and since $M \leq N$ and $\boldsymbol{\Phi}\left(\boldsymbol{D}_{P}+\boldsymbol{C}_{P} \boldsymbol{B}_{P}\right) \boldsymbol{u}^{*}=\boldsymbol{r}^{\Phi}$

$$
\begin{aligned}
\lambda= & -\left(\boldsymbol { \Phi } ( \boldsymbol { D } _ { P } + \boldsymbol { C } _ { P } \boldsymbol { B } _ { P } ) \left(\left(\boldsymbol{D}_{H}+\boldsymbol{C}_{H} \boldsymbol{B}_{H}\right)^{\top}\right.\right. \\
& \left.\left.\left(\boldsymbol{D}_{H}+\boldsymbol{C}_{H} \boldsymbol{B}_{H}\right)\right)^{-1}\left(\boldsymbol{\Phi}\left(\boldsymbol{D}_{P}+\boldsymbol{C}_{P} \boldsymbol{B}_{P}\right)\right)^{\top}\right)^{-1} \boldsymbol{r}^{\Phi} \\
\Rightarrow \hat{\boldsymbol{u}}= & \left(\left(\boldsymbol{D}_{H}+\boldsymbol{C}_{H} \boldsymbol{B}_{H}\right)^{\top}\left(\boldsymbol{D}_{H}+\boldsymbol{C}_{H} \boldsymbol{B}_{H}\right)\right)^{-1} \\
& \times\left(\boldsymbol{\Phi}\left(\boldsymbol{D}_{P}+\boldsymbol{C}_{P} \boldsymbol{B}_{P}\right)\right)^{\top} \\
& \times\left(\boldsymbol { \Phi } ( \boldsymbol { D } _ { P } + \boldsymbol { C } _ { P } \boldsymbol { B } _ { P } ) \left(\left(\boldsymbol{D}_{H}+\boldsymbol{C}_{H} \boldsymbol{B}_{H}\right)^{\top}\right.\right. \\
& \left.\left.\times\left(\boldsymbol{D}_{H}+\boldsymbol{C}_{H} \boldsymbol{B}_{H}\right)\right)^{-1}\left(\boldsymbol{\Phi}\left(\boldsymbol{D}_{P}+\boldsymbol{C}_{P} \boldsymbol{B}_{P}\right)\right)^{\top}\right)^{-1} \boldsymbol{r}^{\Phi} .
\end{aligned}
$$

215

$$
\begin{aligned}
& \text { This can be expressed as } \hat{\boldsymbol{u}}=A^{\dagger} \boldsymbol{r}^{\Phi} \text { where } \\
& A^{\dagger}:=X A^{\top}\left(A X A^{\top}\right)^{-1} \text { with } X=\left(\left(\boldsymbol{D}_{H}+\right.\right. \\
& \left.\left.\boldsymbol{C}_{H} \boldsymbol{B}_{H}\right)^{\top}\left(\boldsymbol{D}_{H}+\boldsymbol{C}_{H} \boldsymbol{B}_{H}\right)\right)^{-1} \text {. }
\end{aligned}
$$

Next consider the update (35) which can be written as

$$
\begin{aligned}
\boldsymbol{v}(k+1) & =(\boldsymbol{\Phi} \boldsymbol{P} \boldsymbol{F}) \boldsymbol{u}(k+1)=\boldsymbol{\Phi} \boldsymbol{D}_{P} \boldsymbol{D}_{F} u(k+1) \\
& +\boldsymbol{\Phi}\left(\boldsymbol{D}_{P} \boldsymbol{C}_{F} \boldsymbol{B}_{F}+\boldsymbol{C}_{P} \boldsymbol{B}_{P} \boldsymbol{D}_{F}\right) \boldsymbol{u}(k) \\
& +\boldsymbol{\Phi} \underbrace{\left(\boldsymbol{C}_{P} \boldsymbol{B}_{P} \boldsymbol{C}_{F} \boldsymbol{B}_{F}\right.}_{=0} \boldsymbol{u}(k-1)
\end{aligned}
$$

where the constraint $n_{F}+n_{P} \leq N$ ensures $\boldsymbol{C}_{P} \boldsymbol{B}_{P} \boldsymbol{C}_{F} \boldsymbol{B}_{F}=0$. After considerable manipulation it is possible to write the recurrence relation

$$
\begin{aligned}
\boldsymbol{u}(k+1)= & \boldsymbol{u}(k)+\beta v(k) \\
= & \left(I+\beta \boldsymbol{\Phi} \boldsymbol{D}_{P} \alpha_{2}\right)^{-1} \\
& \times\left\{\left(\alpha_{1}+\alpha_{2}+\alpha_{3}\right) \boldsymbol{r}+\left(I-\beta \boldsymbol{\Phi} \boldsymbol{D}_{P} \alpha_{1}\right.\right. \\
& \left.-\beta \boldsymbol{\Phi} \boldsymbol{C}_{P} \boldsymbol{B}_{P} \alpha_{2}\right) \boldsymbol{u}(k) \\
& \left.-\beta\left(\boldsymbol{\Phi} \boldsymbol{D}_{P} \alpha_{3}+\boldsymbol{\Phi} \boldsymbol{C}_{P} \boldsymbol{B}_{P} \alpha_{1}\right) \boldsymbol{u}(k-1)\right\}
\end{aligned}
$$

where

$$
\begin{aligned}
\alpha_{1} & =\boldsymbol{D}_{F}\left(\boldsymbol{\Phi} \boldsymbol{D}_{P} \boldsymbol{D}_{F}\right)^{\top}+\boldsymbol{C}_{F} \boldsymbol{B}_{F}\left(\boldsymbol{\Phi} \boldsymbol{D}_{P} \boldsymbol{C}_{F} \boldsymbol{B}_{F}\right. \\
& \left.+\boldsymbol{\Phi} \boldsymbol{C}_{P} \boldsymbol{B}_{P} \boldsymbol{D}_{F}\right)^{\top} \\
\alpha_{2} & =\boldsymbol{D}_{F}\left(\boldsymbol{\Phi} \boldsymbol{D}_{P} \boldsymbol{C}_{F} \boldsymbol{B}_{F}+\boldsymbol{\Phi} \boldsymbol{C}_{P} \boldsymbol{B}_{P} \boldsymbol{D}_{F}\right)^{\top} \\
\alpha_{3} & =\boldsymbol{C}_{F} \boldsymbol{B}_{F}\left(\boldsymbol{\Phi} \boldsymbol{D}_{P} \boldsymbol{D}_{F}\right)^{\top} .
\end{aligned}
$$

Further manipulation, and application of block matrix inverse relations, see [17], gives

$$
\begin{aligned}
\boldsymbol{u}_{\infty}= & {\left[\boldsymbol{\Phi}\left(\boldsymbol{D}_{P}+\boldsymbol{C}_{P} \boldsymbol{B}_{P}\right)\left(\left(\boldsymbol{D}_{H}+\boldsymbol{C}_{H} \boldsymbol{B}_{H}\right)^{\top}\left(\boldsymbol{D}_{H}+\boldsymbol{C}_{H} \boldsymbol{B}_{H}\right)\right)^{-1}\right.} \\
& \left.\times\left(\boldsymbol{\Phi}\left(\boldsymbol{D}_{P}+\boldsymbol{C}_{P} \boldsymbol{B}_{P}\right)\right)^{\top}\right]^{-1}\left(\left(\boldsymbol{D}_{H}+\boldsymbol{C}_{H} \boldsymbol{B}_{H}\right)^{\top}\right. \\
& \left.\times\left(\boldsymbol{D}_{H}+\boldsymbol{C}_{H} \boldsymbol{B}_{H}\right)\right)^{-1}\left(\boldsymbol{\Phi}\left(\boldsymbol{D}_{P}+\boldsymbol{C}_{P} \boldsymbol{B}_{P}\right)\right)^{\top} \boldsymbol{r}^{\Phi}
\end{aligned}
$$

which equates to 41 as required.

Although causal, update (35) cannot be applied in practice since calculation of $\boldsymbol{u}_{\boldsymbol{k}+\mathbf{1}}$ requires knowledge of the whole of $\boldsymbol{e}_{\boldsymbol{k}+\mathbf{1}}$. Therefore (35) must now be converted to an along-the-trial form.

Proposition 1. Update (35) has equivalent unlifted form

$$
\begin{aligned}
& u((k+1) N+i)=u(k N+i)+ \\
& \beta\left(\sum_{j=\underline{i}_{f}}^{M} \psi\left(i_{j}-i-N\right) e\left((k-1) N+i_{j}\right)+\right. \\
& \sum_{j=\bar{i}_{f}}^{M} \psi\left(i_{j}-i\right) e\left(k N+i_{j}\right)+ \\
& \left.\sum_{j=1}^{\bar{i}} \psi\left(N+i_{j}-i\right) e\left((k+1) N+i_{j}\right)\right)
\end{aligned}
$$


where $\bar{i}=\max _{j \geq 0}\left\{i_{j} \mid i_{j} \leq i, i_{0}=0\right\}, \bar{i}_{f}=$ $\min _{j \geq 1}\left\{i_{j} \mid i_{j} \geq i-n_{F}\right\}$ and $\underline{i}_{f}=\min _{j \geq 1}\left\{i_{j} \mid i_{j} \geq\right.$ $\left.i-n_{F}+N, i_{m+1}=2 N\right\}$. Function $\psi(i):=$ $\sum_{a=-n_{F}}^{n_{F}} \sum_{j=0}^{n_{F}} f(j) f(a+j) p(i+a)$ where $f(i)=$ $C_{F} A_{F}^{i} B_{F}, p(i)=C_{P} A_{P}^{i} B_{P}$, are the impulse responses of $F(q)$ and $P(q)$ respectively.

Proof. Since $F(q)$ is causal, we write $v(q)=$ $F(q) w(q)$ in the FIR convolution form

$$
v(i)=\sum_{j=0}^{n_{F}} f(j) w(i-j)
$$

and similarly $w(q)=F(q)^{*} x(q)$ can be written as

$$
w(i)=\sum_{r=0}^{n_{F}} f(r) x(i+r)
$$

and it follows that $\boldsymbol{v}=\boldsymbol{F}(\boldsymbol{q}) \boldsymbol{F}^{*}(\boldsymbol{q}) \boldsymbol{x}$ corresponds to the unlifted update

$$
\begin{aligned}
v(i) & =\sum_{j=0}^{n_{F}} f(j) \sum_{r=0}^{n_{F}} f(r) x(i-j+r) \\
& =\sum_{j=0}^{n_{F}} \sum_{a=-j}^{n_{F}-j} f(j) f(a+j) x(i+a) \\
& =\sum_{a=-n_{f}} \underbrace{\left\{\sum_{j=0}^{n_{F}} f(j) f(a+j)\right\}}_{:=h(a)} x(i+a)
\end{aligned}
$$

where $h(a)$ is defined over $a \in[-n, n]$. It can be shown that the operator $\boldsymbol{x}=\boldsymbol{\Phi} \boldsymbol{P}^{*}(\boldsymbol{q}) \boldsymbol{e}$ has form,

$$
\begin{aligned}
\boldsymbol{x}(k) & =\left(\overline{\boldsymbol{\Phi}} \boldsymbol{D}_{P}\right)^{\top} \overline{\boldsymbol{\Phi}} \boldsymbol{e}(k)+ \\
& \sum_{i=1}^{\infty}\left\{\left(\boldsymbol{C}_{P}\left(\boldsymbol{A}_{P}\right)^{i-1} \boldsymbol{B}_{P}\right)^{\top} \overline{\boldsymbol{\Phi}}^{\top} \overline{\boldsymbol{\Phi}} \boldsymbol{e}(k+i)\right\}
\end{aligned}
$$

and since 36 implies 33 holds, this simplifies to

$$
\boldsymbol{x}(k)=\left(\overline{\mathbf{\Phi}} \boldsymbol{D}_{P}\right)^{\top} \overline{\boldsymbol{\Phi}} \boldsymbol{e}(k)+\boldsymbol{B}_{P}^{\top}\left(\overline{\mathbf{\Phi}} \boldsymbol{C}_{P}\right)^{\top} \overline{\boldsymbol{\Phi}} \boldsymbol{e}(k+1)
$$

which has the unlifted form

$$
\begin{aligned}
x(k N+i) & =\sum_{j=1}^{\bar{i}-n_{f}} p\left(N+i_{j}-i\right) e\left((k+1) N+i_{j}\right) \\
& +\sum_{j=\bar{i}+1}^{M} p\left(i_{j}-i\right) e\left(k N+i_{j}\right) .
\end{aligned}
$$

where $\bar{i}=\max _{j} i_{j} \leq i$ is the most recent point-topoint index.
Substituting 51 into 48 and then into 35 gives rise to the update

$$
\begin{aligned}
& u((k+1) N+i)=u(k N+i) \\
& +\beta\left(\sum_{j=1}^{\bar{i}-n_{f}} \sum_{a=-n_{f}}^{n_{f}} h(a) p\left(N+i-i_{j}+a\right) e\left((k+1) N+i_{j}\right)\right. \\
& \left.+\sum_{j=\bar{i}+1}^{M} \sum_{a=-n_{f}}^{n_{f}} h(a) p\left(i_{j}-i+a\right) e\left(k N+i_{j}\right)\right)
\end{aligned}
$$

Partitioning the sum into separate cycles, $k-1, k$, $k+1$, yields the form 45 .

Remark 1. Selecting $F=I$ in Theorem 4 reduces update (35) to the simplified form of (34). In this case optimal control problem (38) then simplifies to

$$
\hat{\boldsymbol{u}}:=\min _{\boldsymbol{u}}\|\boldsymbol{u}\|^{2} \quad \text { such that } \quad \boldsymbol{r}^{\Phi}=\boldsymbol{\Phi} \boldsymbol{P}(\boldsymbol{q}) \boldsymbol{u}
$$

This is the minimum control effort solution to the tracking problem, and is of special significance to the current application since it has been proposed as a solution to human motor control and therefore can be expected to approximate natural motion.

Remark 2. Selecting $F=I$ in Proposition 1 reduces the unlifted update form (45) to

$$
\begin{aligned}
u((k+1) N+i) & =u(k N+i) \\
& +\beta\left(\sum_{j=1}^{\bar{i}} C A^{N-i+i_{j}} B e\left((k+1) N+i_{j}\right)\right. \\
& \left.+\sum_{j=\bar{i}+1}^{M} C A^{i-i_{j}} B e\left(k N+i_{j}\right)\right)
\end{aligned}
$$

Remark 3. Other choices of filter F can lead to a more accurate representation of human motor ples for the assistance of human motor function is: $H(q)=\frac{1}{q^{n}}, n \geq 1$ (i.e. minimisation of velocity, acceleration, jerk norms etc.). These correspond to $F(q)=q^{n}, n \geq 1$. Here $H$ and $F$ are the unlifted 245 transfer function versions corresponding to $\boldsymbol{H}$ and $\boldsymbol{F}$ in Theorem 4. The associated impulse responses $h$ and $f$ are, however, infinite and hence cannot satisfy the requirement $n_{F}+n_{P} \leq N$. It is therefore necessary to replace them with suitable approx250 imations, or to cut off the update when it becomes non-causal.

\section{Experimental Application to Drop-Foot}

Although widely applicable in other domains, the motivation for the point-to-point $\mathrm{RC}$ framework 

experimentally applied to assess its performance to improve accuracy, reduce control effort and achieve normal motion.

\subsection{Hardware}

To apply the electrical pulses that induce movement in the muscle, electrode pads need to be placed on the skin. To find the best location, the fabric based electrode array developed in [18. was used. This was placed on the shank, and an ankle goniometer was attached around the joint within a specially designed sock. The identification and control system was implemented using a myRIO (National Instruments) microprocessor which outputs a pulsewidth modulated signal to drive the FES. This 270 signal was amplified by a stimulator unit [19] to a level which achieves muscle contraction, and outputted to the electrode array. For each particpant, the FES current amplitude was tuned to be within a comfortable region at the beginning of the experi-

275 ment. The pulsewidth was then the controlled variable and was restricted to $0 \leq u \leq 300$. A sampling frequency of $40 \mathrm{~Hz}$ was used by the control system.

\subsection{Model Identification}

The plant model $P$ of equation (1) represents the response of the ankle to electrical stimulation, and must be identified. The best stimulation position was first found by applying FES to each pad in turn and selecting the pad which produces the maximum dorsiflexsion and minimum twitch response and roll. This is a similar approach to that reported in 20 .

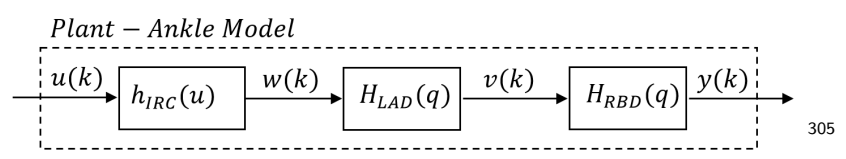

Figure 4: Stimulated ankle system; static non-linearity, linear activation dynamics and rigid body dynamic model.

Once a pad was selected, the dynamics relating the applied FES and the resulting angular move- 310 ment was identified. These comprise the isometric recruitment curve (IRC), the muscle linear activation dynamics (LAD), and the rigid body dynamics (RBD). The IRC was acquired using a slow (approximately steady state) ascending and descending 315 (triangle) signal, as described in [21. To perform 295 a linearising action, a monotonic realisation of the
IRC is required which was fitted using a 3 parameter spline function. The inverse of this function was then applied by the controller to cancel the nonlinearity, as shown in Figure 5. The remaining dynamics were then identified by applying sufficiently exciting FES signals and employing least mean squared parameter identification. From this the state-space model parameters were obtained.

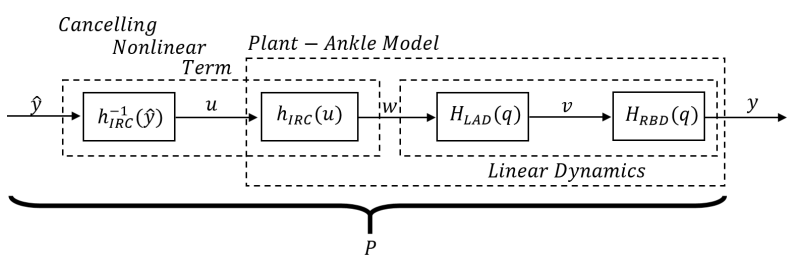

Figure 5: Stimulated ankle system with linearising action.

\subsection{Gait Cycle}

Most medical texts express gait cycle positions as a percentage, to avoid issues of varying speed and stride lengths, the same approach will be taken here. Gait is commonly split into stance $(60 \%$ of motion) and swing (40\% of motion). This can then be further partitioned into 8 distinct sections 22 . By amalgamating experimental results from 23 and 24 a full human gait cycle is shown in Figure 6. Although gait periods vary, an average of 2 seconds is commonly assumed. From this cycle, five key points have been extracted that correspond to extremes of motion and key transitions. These will be the points tracked by point-to-point $\mathrm{RC}$. The $M=5$ tracked points in Figure 6 are

$$
i_{1}=5 \%, i_{2}=25 \%, i_{3}=45 \%, i_{4}=63 \%, i_{5}=84 \% .
$$

These correspond to the following samples,

$$
i_{1}=4, i_{2}=20, i_{3}=36, i_{4}=51, i_{5}=68 .
$$

\subsection{Methodology}

Experimental trials with healthy individuals were undertaken to evaluate the effectiveness of the designed RC schemes. Following ethical approval (ERGO/FPSE/47517), five participants were recruited for this study. Participant 1 and 5 had experience/partaken in FES studies before, while participants 2, 3 and 4 had not. The participants are asked to sit on an elevated stool, thus leaving the leg able to swing freely.

To limit the effects of fatigue there was a 20 minute break between each test, allowing the muscles to recover [25]. To limit the impact of model 


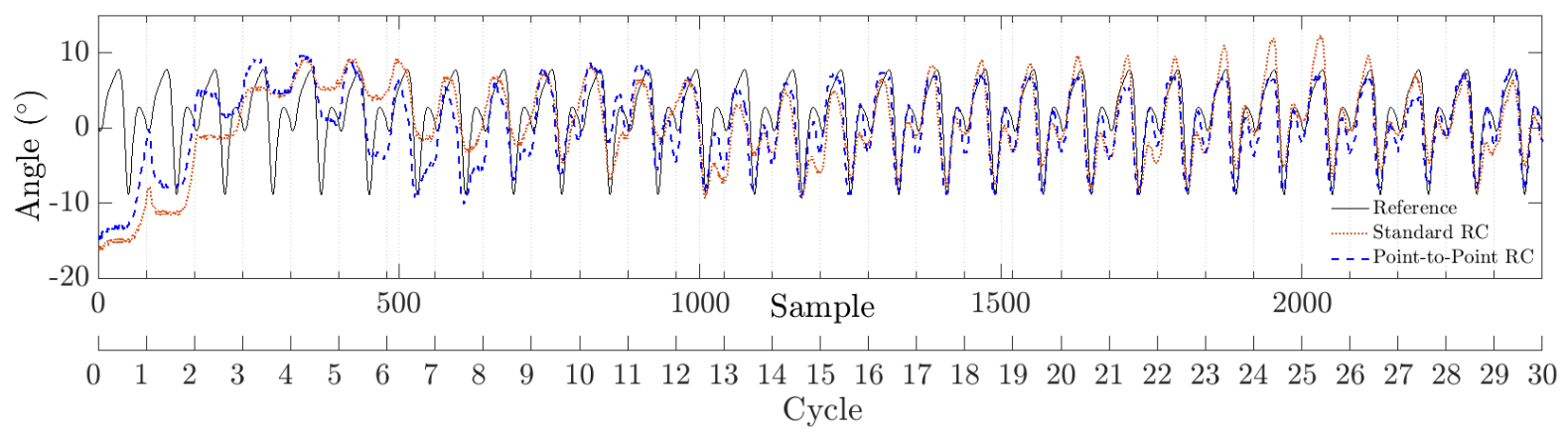

Figure 7: Typical outputs of both control schemes.

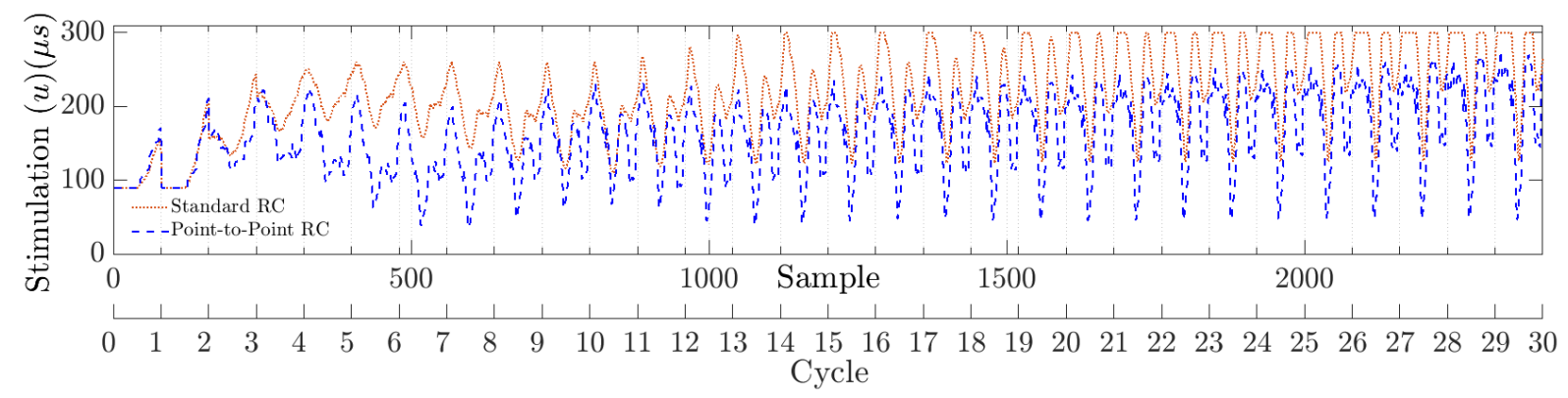

Figure 8: Inputs required to produce the tracking outputs.

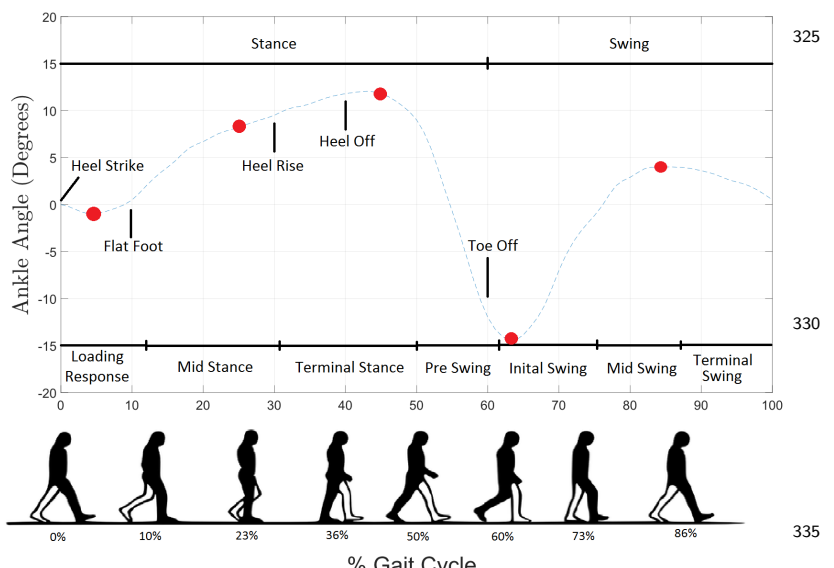

Figure 6: Standardised gait cycle, with gait events/phases labelled. The red dots correspond to the point-to-point tracking positions.

uncertainty, all tests with a participant were conducted in a single session, using the same initial

\section{Results}

Tests were performed using both tradiational $\mathrm{RC}$ (i.e. tracking a reference defined by all sample instances) and point-to-point RC to compare their

ability to produce normal gait motion.

\subsection{Converged Output}

The traditional and point-to-point $\mathrm{RC}$ are implemented with (4) and (54), both of which use the gradient algorithm put forward in Lemma 3.

Figure 7 shows the results from a representative trial from participant 1 . The tracking can be seen to converge faster for the point-to-point scheme as well as maintain tracking, despite the presence of noise, exogenous disturbance and fatigue. This confirms Theorem 11 and the convergence criteria in Theorem 2. For completeness, Figure 8 shows the stimulation $u$ which produced Figure 7

\subsection{Error Norm}

Figure 9 shows the repeatability of each control scheme. Each scheme is repeated 3 times with the same $\beta$ value, $80 \%$. The percentage denote how the selected value differ from the value of $\beta$ which produces fastest convergence based on their nominal plant model, i.e. the nominal value

$$
\frac{1}{\sup _{\omega \in[0, \pi]}\left|P\left(e^{j \omega}\right)\right|^{2}} .
$$


The points of subset $M$ are used to compute the 340 error norm, with different starting values due to small variations in starting angle. The point-topoint controller can be seen to converge faster than standard repetitive control as well as demonstrating repeatable characteristics despite involuntary witch response and fatigue. It is also observed that the point-to-point has little to no oscillation visible in the norm suggesting improved robustness to model uncertainty.

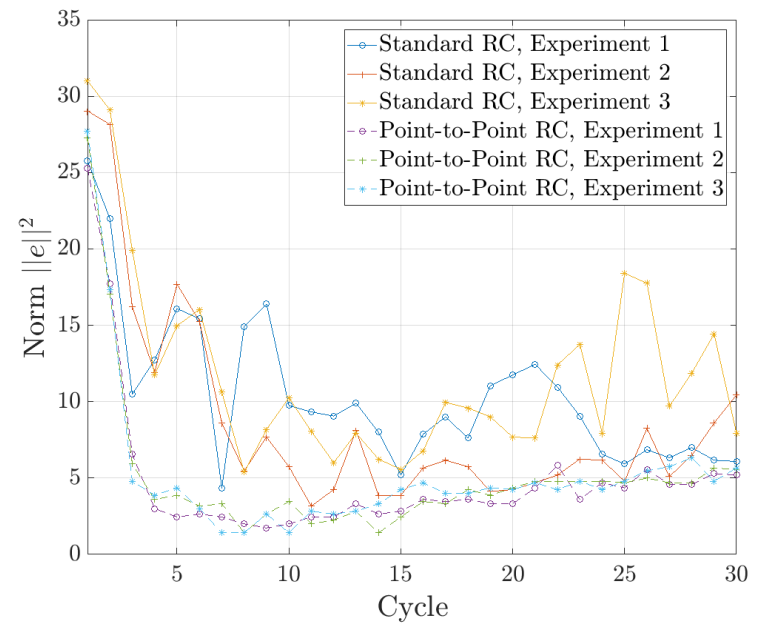

Figure 9: The effect of altering learning rate on convergence.

\subsection{Control Effort}

The amount of control effort is of interest as it directly leads to fatigue and possible discomfort. Figure 10 shows the control effort norm for each cycle $\left(\|u\|^{2}\right)$ to achieve the tracking motion. Point- 385 to-point $\mathrm{RC}$ can be seen to require less energy to achieve the same task as the standard RC. The stimulation increases with time as it acts against the effect of fatigue. This can also been seen in Figure 8 and 7. After $30-40$ cycles the control ef- ${ }^{390}$ fort level off to a fixed value as the effect of fatigue is fully realised.

Figure 8, shows the control effort applied during each cycle of both schemes. The traditional RC can be seen to use more stimulation as it tries to 395 achieve perfect tracking of the full reference. Due the nonlinear relationship between control effort and angular position, the resulting input signals can appear very different.

It is important to note that both repetitive con- 400 trol schemes lead to a stimulation input that is

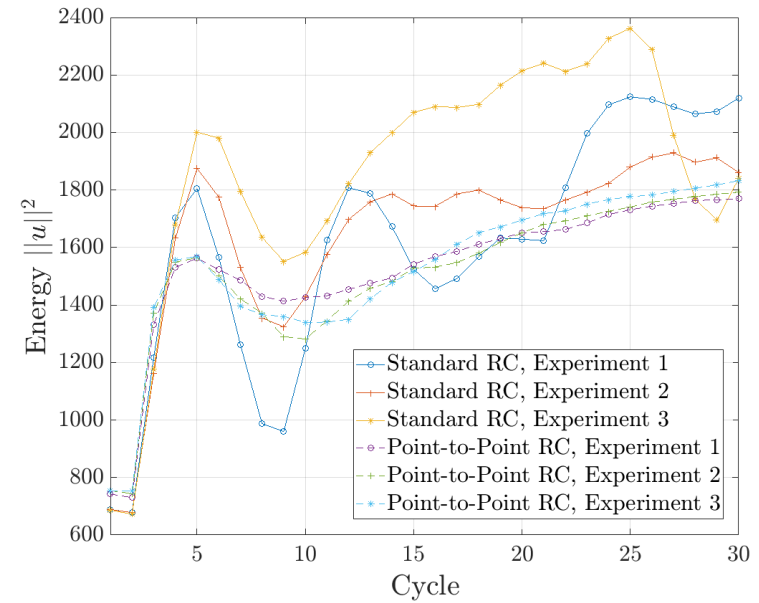

Figure 10: Control effort comparison for both schemes and varying learning rates.

from day to day use, without any changes to the FES parametrization and reference trajectory used. This illustrates the advantage of optimising a cost as opposed to tracking a homogenous gait profile.

\subsection{Comparison}

Table 1 contains data gathered from all participants using only $80 \%$ of the optimal $\beta$ values computed using their nominal plant model. It compares key parameters for both the traditional $\mathrm{RC}$ and point-to-point RC.

The point-to-point $\mathrm{RC}$ and traditional RC controllers achieve similar tracking of the subset of points $M$. The total control effort for the pointto-point scheme is lower in the majority of cases, reflecting the minimum energy property of Theorem 4. Comparing entire signals, both controllers achieve excellent tracking, despite the point-topoint controller having $\frac{1}{16}$ of the points to track, justifying the use of the gradient algorithm in Lemma 3 and the minimum energy filter from 4 The point-to-point $\mathrm{RC}$ can also be seen to converge below a $10 \%$ and $5 \%$ error norm faster, where this was achievable.

Table 2 contains the RMSE (degree) of the last 5 cycles of the recorded data. The mean and standard deviation for the 5 participants using traditional $\mathrm{RC}$ are $2.56^{\circ} \pm 0.76^{\circ}$ and for point-to-point $\mathrm{RC}$ are $2.99^{\circ} \pm 0.99^{\circ}$. This illustrates that in all cases the RMSE falls within the natural variance of gait [10].

$\mathrm{RC}$ and point-to-point $\mathrm{RC}$ have been shown to achieve better tracking than neural networks $\left(8.11^{\circ}, 7.81^{\circ}\right.$ and $\left.5.59^{\circ}\right)$, neural network + PID 


\begin{tabular}{|c|c|c|c|c|c|c|c|c|}
\hline \multirow[t]{2}{*}{ Participant } & \multicolumn{2}{|c|}{$\begin{array}{l}\text { Average P-to-P Norm } \\
\left(\frac{1}{5} \sum_{n=26}^{30}\left\|\boldsymbol{\Phi} e_{n}\right\|^{2}\right)\end{array}$} & \multicolumn{2}{|c|}{$\begin{array}{l}\text { Control Effort } \\
\left(\sum_{n=0}^{30}\left\|u_{n}\right\|^{2}\right)\end{array}$} & \multicolumn{2}{|c|}{$\begin{array}{c}\text { Average Norm } \\
\text { of Full Cycle } \\
\left(\frac{1}{5} \sum_{n=26}^{30}\left\|e_{n}\right\|^{2}\right) \\
\text { [Average Degree Error] }\end{array}$} & \multicolumn{2}{|c|}{$\begin{array}{c}\text { Cycles to } \\
10 \%[5 \%] \text { Error } \\
\text { Norm }\left(\|\mathbf{\Phi} e\|^{2}\right)\end{array}$} \\
\hline & $\mathrm{RC}$ & P-to-P RC & $\mathrm{RC}$ & P-to-P RC & $\mathrm{RC}$ & P-to-P RC & $\mathrm{RC}$ & P-to-P RC \\
\hline 1 & 45.13 & 40.16 & 7519 & 43598 & $43.72\left[1.8^{\circ}\right]$ & $41.1\left[1.6^{\circ}\right]$ & $9[20]$ & $6[12]$ \\
\hline 2 & 89.8 & 85 & 895 & 48 & $63.1\left[2.6^{\circ}\right]$ & $110.39\left[4.2^{\circ}\right]$ & $16[\mathrm{~N} / \mathrm{A}]$ & $12[\mathrm{~N} / \mathrm{A}]$ \\
\hline 3 & 75.9 & 77 & 49745 & 46416 & $56.2\left[1.93^{\circ}\right]$ & $78.3\left[2.6^{\circ}\right]$ & $10[15]$ & $7[10]$ \\
\hline 4 & 34.88 & 39.28 & 34691 & 50948 & $13.1\left[1.28^{\circ}\right]$ & $17.67\left[1.49^{\circ}\right]$ & $4[8]$ & $4[7]$ \\
\hline 5 & 47.1 & 48.8 & 43779 & 44501 & $66.65^{\circ}\left[2.33^{\circ}\right]$ & $70.2\left[2.48^{\circ}\right]$ & $7[10]$ & 5 [10] \\
\hline
\end{tabular}

Table 1: Comparison Table

\begin{tabular}{ccc}
\hline \multirow{2}{*}{ Participant } & \multicolumn{2}{c}{$\mathrm{RMSE}\left(\sqrt{\frac{e^{2}}{5 T_{s}}}\right)$} \\
\cline { 2 - 3 } & $\mathrm{RC}$ & P-to-P RC \\
\hline 1 & $2.19^{\circ}$ & $2.06^{\circ}$ \\
2 & $3.15^{\circ}$ & $5.52^{\circ}$ \\
3 & $2.81^{\circ}$ & $3.91^{\circ}$ \\
4 & $1.57^{\circ}$ & $1.97^{\circ}$ \\
5 & $3.32^{\circ}$ & $3.51^{\circ}$ \\
\hline
\end{tabular}

Table 2: Root mean square degree error of the last 5 recorded cycles of both control schemes

$\left(5.57^{\circ}, 4.83^{\circ}\right.$ and $\left.5.05^{\circ}\right)$, neural networks + fuzzy $\operatorname{logic}\left(4.07^{\circ}, 3.75^{\circ}\right.$ and $\left.4.19^{\circ}\right)$ [7, model-based open-

loop $\left(8.2^{\circ} \pm 2.3^{\circ}, 15.1^{\circ} \pm 1.9^{\circ}\right)$, feedback $\left(4.6^{\circ} \pm\right.$ $0.7^{\circ}, 7.3^{\circ} \pm 2.4^{\circ}$ ) feed-forward feedback hybrid $\left(3.4^{\circ} \pm 0.3^{\circ}, 5.7^{\circ} \pm 2.6^{\circ}\right)[26]$ and similar to adaptive control, healthy $3.2^{\circ} \pm 1.2^{\circ}$, stroke $3.4^{\circ} \pm 0.2^{\circ}[8]{ }_{440}$ noting simple references were used in this case. The

${ }_{410} \mathrm{RC}$ controllers have achieved this with no time-lag issues that effected the feedback based controllers. The point-to-point RC controller also used a frac- ${ }^{445}$ tion of the measurement data.

\section{Conclusion}

415

This paper has developed a framework that increases the flexibility of RC and has potential utilisation in applications that demand fast convergence, optimal input signals and reduced sensor information. Experimental results have shown point-

${ }_{420}$ to-point $\mathrm{RC}$ to be more robust to model uncertainty than traditional RC, producing faster convergence whilst using/storing less data. It has also been shown that less stimulation is required to achieve tracking facilitated by the reduction in points to
425 track. The controller has shown it is able to successfully maintain tracking despite fatigue by increasing the control effort with time. The controllers put forward have also been compared to other controllers, where superior tracking has been observed for the $\mathrm{RC}$ and point-to-point RC schemes.

Future work will investgate alternative filter selection, such as the minimum jerk cost function proposed in [27. The system will then be evaulated in a clinical trial with stroke patients.

\section{Acknowledgements}

This work was supported by Engineering and Physical Sciences Research Council grant $\mathrm{EP} / \mathrm{M} 026388 / 1$.

[1] C. L. Barrett, P. N. Taylor, The effects of the odstock drop foot stimulator on perceived quality of life for people with stroke and multiple sclerosis, Neuromodulation 13 (1) (2010) 58-64.

[2] E. W. Peterson, Fear of falling and associated activity curtailing among middle aged and older adults with multiple scleosis, Multiple Sclerosis 13 (2007) 11681175 .

[3] J. E. Esnouf, P. N. Taylor, G. E. Mann, C. L. Barrett, Impact on activities of daily living using a functional electrical stimulation device to improve dropped foot in people with multiple sclerosis, measured by the canadian occupational performance measure, Mult Scler 16 (2010) 1141-1147.

[4] A. Roche, G. O. Laighin, S. Coote, Surface-applied functional electrical stimulation for orthotic and therapeutic treatment of drop-foot after stroke: a systematic review, Physical Therapy Reviews 14 (2) (2009) 63-80.

[5] A. Kostov, R. B. Stein, D. Popovic, W. W. Armstrong, Improved methods for control of fes for locomotion, IFAC Proceedings Volumes 27 (1) (1994) $445-450$.

[6] L. P. Kenney, B. W. Heller, A. Barker, M. L. Reeves, J., T. R. Good, G., N. Sha, S. Prenton, A. Liu, D. Howard, A review of the design and clinical evaluation of the shefstim array-based functional electrical stimulation 
system, Medical Engineering \& Physics 38 (11) (2016) $1159-1165$.

[7] Y. Chen, S. Chen, W. Chen, C. Hsiao, T. Kuo, J. Lai, Neural network and fuzzy control in fes-assisted locomotion for the hemiplegic, Journal of Medical Engineering \& Technology 28 (1) (2004) 32-38.

[8] H.-R. Kobravi, A. Erfanian, Decentralized adaptive ro- 535 bust control based on sliding mode and nonlinear compensator for the control of ankle movement using functional electrical stimulation of agonist-antagonist muscles, Journal of Neural Engineering 6 (4) (2009) 046007.

[9] N. Negrd, Controlled FES-assisted gait training for 540 hemiplegic stroke patients based on inertial sensors, Ph.D. thesis, TU Berlin (2009).

[10] T. Seel, C. Werner, J. Raisch, T. Schauer, Iterative learning control of a drop foot neuroprosthesis generating physiological foot motion in paretic gait by auto- 545 matic feedback control., Control Engineering Practice 48 (3) (2016) 87-97.

[11] G. Pipeleers, K. Moore, Unified analysis of iterative learning and repetitive controllers in trial domain, IEEE Transactions on Automatic Control 59 (4) (2014) 953- 550 965.

[12] E. Copur, C. Freeman, B. Chu, D. Laila, Repetitive control of electrical stimulation for tremor suppression, IEEE Transactions on Control Systems Technology 27 (2) (2017) 540-552.

[13] Y. Han, D. Owens, B. Chu, Linear optimal multiperiodic repetitive control - a low order controller scheme, An International Journal of Optimization and Control: Theories \& Applications 2 (1) (2011) 1-16.

[14] R. W. Longman, Iterative learning control and repetitive control for engineering practice, International Journal of Control 73 (2000) 930-954.

[15] J. J. Hätönen, C. T. Freeman, D. H. Owens, P. L. Lewin, E. Rogers, A gradient-based repetitive control algorithm combining ILC and pole placement, Euro. J. of Control 12 (3) (2006) 278-292.

[16] J. J. Hätönen, C. T. Freeman, D. H. Owens, P. L. Lewin, E. Rogers, Robustness analysis of a gradientbased repetitive algorithm for discrete-time systems, in: 43rd IEEE Conference on Decision and Control, Vol. 2, 2004, pp. 1301-1306.

[17] T. Lu, S.-H. Shiou, Inverses of $2 \times 2$ block matrices, Computers and Mathematics with Applications 43 (2002) 119-129.

[18] K. Yang, C. Freeman, R. Torah, S. Beeby, J. Tudor, Screen printed fabric electrode array for wearable functional electrical stimulation, Sensors and Actuators A: Physical 213 (2014) 108-115.

[19] M. Ilic, D. Vasiljevic, D. B. Popovic, A programmable electronic stimulator for fes systems, IEEE Transactions on Rehabilitation Engineering 2 (4) (1994) 234-239.

[20] S. Prenton, L. Kenney, C. Stapleton, G. Cooper, M. Reeves, B. Heller, M. Sobuh, A. Barker, J. Healey, T. Good, S. B. Thies, T. Howard, D.and Williamson, Feasibility study of a take-home array-based functional electrical stimulation system with automated setup for current functional electrical stimulation users with footdrop, Archives of Physical Medicine and Rehabilitation 95 (10) (2014) 1870-1877.

[21] F. Le, I. Markovsky, C. T. Freeman, E. Rogers, Identification of electrically stimulated muscle models of stroke patients, Control Engineering Practice 18 (4) (2010) 396 -407 .
[22] H. Zhang, J. Qian, L. Shen, Y. Zhang, Research on healthy subject gait cycle phase at different walking speeds, in: IEEE International Conference on Robotics and Biomimetics, 2012, pp. 1349-1354.

[23] N. Postans, M. Granat, Effect of functional electrical stimulation, applied during walking, on gait in spastic cerebral palsy, Developmental Medicine and Child Neurology 47 (1) (2005) 46-52.

[24] R. Neptune, K. Sasaki, Ankle plantar flexor force production is an important determinant of the preferred walk-to-run transition speed, Journal of Experimental Biology 208 (5) (2005) 799-808.

[25] G. G. A. Thrasher, M. Popovic, Reducing muscle fatigue due to functional electrical stimulation using random modulation of stimulation parameters, Artificial Organs 29 (6) (2005) 453-458.

[26] M. Ferrarin, F. Palazzo, R. Riener, J. Quintern, Modelbased control of fes-induced single joint movements, IEEE Transactions on Neural Systems and Rehabilitation Engineering 9 (3) (2001) 245-257.

[27] G. Tack, J. S. Choi, J. H. Yi, C. H. Kim, Relationship between jerk cost function and energy consumption during walking, in: R.Magjarevic, J. H. Nagel (Eds.), World Congress on Medical Physics and Biomedical Engineering 2006, Springer Berlin Heidelberg, Berlin, Heidelberg, 2007, pp. 2917-2918. 